\title{
A Tri-band Second-order Frequency Selective Surface Designing and Analysis
}

\author{
Gao Shan ${ }^{\square}$, ChunYan $\mathrm{Gao}^{2,3}$, HongBin $\mathrm{Pu}^{2}$, ChunLan Chen ${ }^{2}$ \\ ${ }^{\square} \mathrm{Xi}$ an Meteorological Bureau, Xi`an, \\ ${ }^{\square}$ Department of Electronic Engineering, Xi'an University of Technology, Xi'an, \\ Shaanxi Youth Vocational college, Xi`an.
}

\begin{abstract}
In this paper, inspired from the technique of patch-aperture-patch, a novel tri-band frequency selective surface with second-order band-pass response in each operation band is presented. The design is implemented by cascading a two-dimensional periodic array of three square loops and an array of wire grids. The proposed structure composed of three metal and two dielectric layers acts as a spatial dual band microwave filter with large band separation. The predicted FSS has the merits of broadband response, excellent stability for different incident angles, and sharp roll-off at $\mathrm{X}-\mathrm{Ku}$ - and $\mathrm{K}$-band, respectively. The simulation and measurement are carried out and discussed. The measured results agree well with the simulated ones.
\end{abstract}

\section{Introduction}

Frequency selective surfaces have been studied extensively for many years [1]. They are widely used in the design of various microwave devices, such as antenna reflectors, radomes, absorbers, as well as electromagnetic band gap materials [2]. In recent years, with the rapid development of satellite communications, the increasing demand for multi-functional antennas for communications requires complex the FSSs with multi-band performances [3]. Especially, the FSSs with multiple independent transmission bands are desired for increasing the communication capabilities of satellite platforms. Generally, multi-band FSSs are the result of the following techniques: Multi-order resonance of a single layer FSS [4-6], loading lumped elements [7] and fractal elements [2], etc. These designs generally have one resonation in each operating band. In recent years, a set of second-order band-pass FSS were presented. For example, a new technique for designing low profile, second- or third-order band pass FSS utilizing non-resonant elements was proposed [8-9]. The patch-aperture-patch (PAP) FSS was proposed and developed in [10-12]. Such a kind of FSS possesses higher selectivity and flat pass-band in its operating frequencies, but such FSS usually have larger element size. Generally, the actual space is small to an ideal infinite periodicity arrays.

In this paper, a miniaturized tri-band FSS with independent operating bands is presented. Each band has merit of second-order response, namely, there are two transmission poles in each band. The size of unit cell is reduced to $\lambda / 8$. There is a large separation between the two adjacent pass-bands, which are operating at X-, Ka- and $\mathrm{K}$-band, respectively. This design is implemented by cascading a two-dimensional periodic array of three square loops and a layer of wire grids. In what follows, the design procedure, the simulation and measurement results of predicted FSS are presented and discussed.

\section{Structure Design and Operation Principle}

The proposed FSS structure consists three layers separated from one another by two thin dielectric substrates, as shown in Fig.1. For simplicity, only one unit cell is presented. The unit cell in exterior two layers is illustrated in Fig.1 (a). Each cell contains two concentric square loops and one curved loop with different dimensions. The middle layer is metal grids structure as shown in Fig.1 (b). The two dielectric substrates are F4B-2 with relative permittivity $\varepsilon_{r}=2.65$ and loss tangent $\tan \delta=0.001$. All the design parameters are $D=5 \mathrm{~mm}, a=4.18 \mathrm{~mm}, b=3.68 \mathrm{~mm}$, $c=0.90 \mathrm{~mm}, \quad d=0.70 \mathrm{~mm}, \quad e=0.40 \mathrm{~mm}, \quad f=3.70 \mathrm{~mm}$, $w_{1}=0.25 \mathrm{~mm}, w_{2}=0.35 \mathrm{~mm}, w_{3}=0.6 \mathrm{~mm}$. The thickness of the one dielectric substrate is $0.6 \mathrm{~mm}$. 


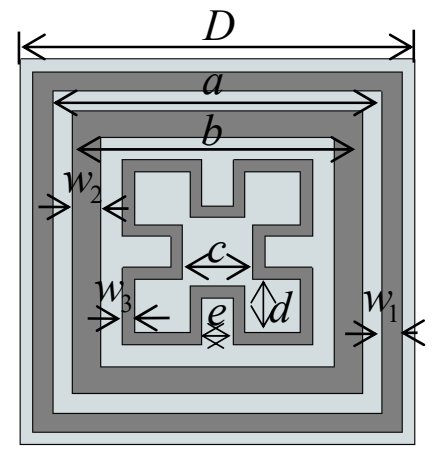

(a) exterior layers

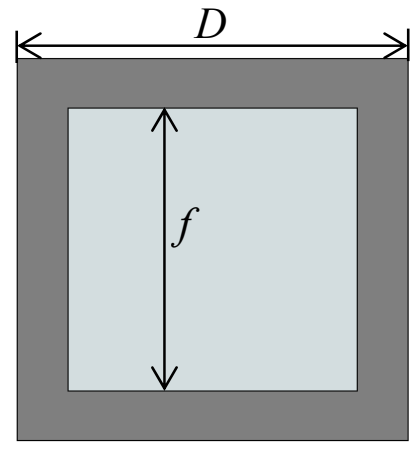

(b) middle layer

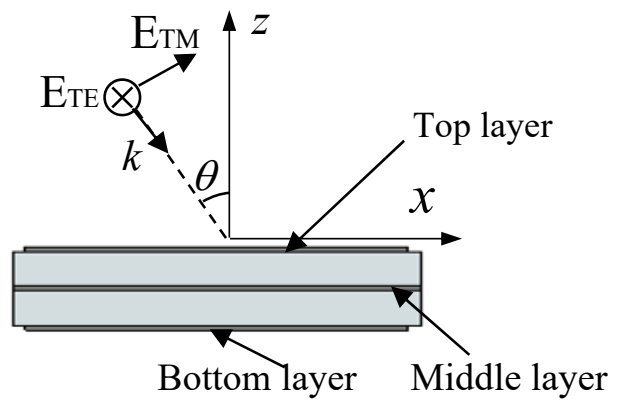

(c) side view

Fig.1. Unit cell in the proposed FSS

Both the square loops and wire grid arrays were extensively studied in the past. Using their equivalent circuit models derived in [13-16], the proposed FSS can be explained using the equivalent circuit of, as shown in Fig. 2 (a), which contains three pairs of shunt serial $L C$ resonators separated from an inductor, $L$, by two short pieces of transmission lines. Each shunt serial $L C$ resonator is composed of two parallel $L C$ circuits, and each LC circuit consists of an inductor $L_{1}\left(L_{2}\right)$ in series with a capacitor $C_{1}\left(C_{2}\right)$. The transmission lines have a characteristics impedance $Z=Z_{0} / \sqrt{\varepsilon}$ and an overall length of $h$, where $\varepsilon r$ is the dielectric constants of the substrate and $Z_{0}=377 \Omega$ is the free space impedance.

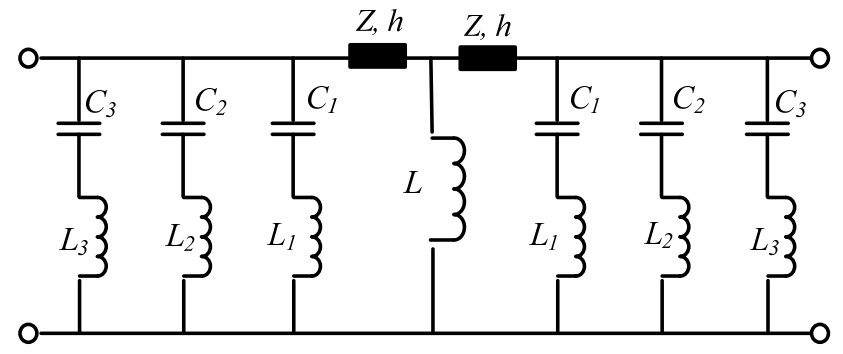

(a)

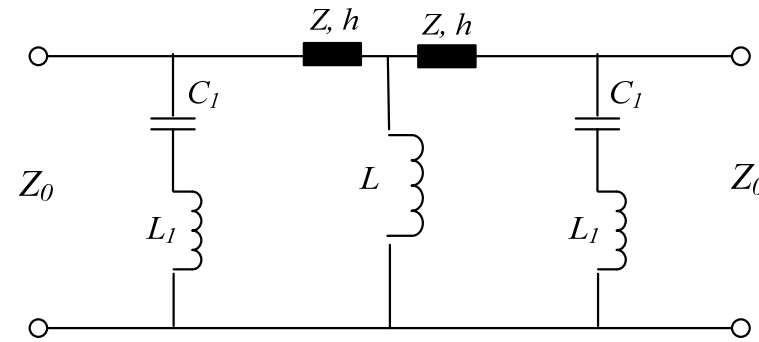

(b)

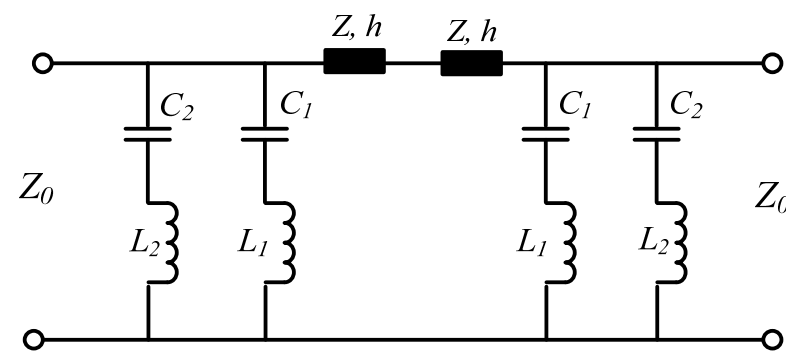

(c)

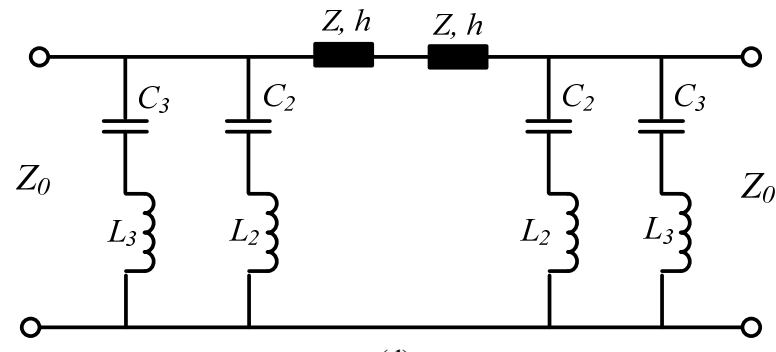

(d)

Fig.2 Equivalent circuit model of the proposed FSS structure

The center frequency of the first pass-band is mainly determined by the values of $L, L_{1}, C_{1}$ and the length of transmission line $h$. Assuming that the three pass bands are sufficiently apart, based on the principle [11], the effect of the serial $L_{2}-C_{2}, L_{3}-C_{3}$ resonators are ignored. This circuit is a classic example of a second-order coupled-resonator band-pass filter, as shown in Fig.1 (a). The frequency $f_{1}$ of the first band of operation can be expressed as follow

$$
f_{1}=1 / 2 \pi \sqrt{\left(C_{1}+\varepsilon_{0} \varepsilon_{r} h / 2\right)\left(L_{1}+L+2 \mu_{0} \mu_{r} h\right)}
$$

Where $\varepsilon_{r}$ and $\mu_{r}$ respectively represent the relative permittivity and the relative permeability of the substrate constituting the transmission line. At the second band of 
operation, the two pairs of shunt circuits composed of serial $L_{1} C_{1}$ resonator and serial $L_{2} C_{2}$ resonator are the dominant resonant and the inductor $L$ is invalidated. So, the filter can be reduced to two parallel resonators separated from one another by a transmission line with an overall length of $2 h$, as shown in Fig. 2 (b). So, the center frequency $f_{2}$ of the second band can be obtained from the formula

$$
f_{2}=\sqrt{\left(C_{1}+C_{2}\right) / C_{1} C_{2}\left(L_{1}+L_{2}\right)} / 2 \pi
$$

Similarly, at the third operating band, the two pairs of shunt circuits composed of serial $L_{2} C_{2}$ resonator and serial $L_{3} C_{3}$ resonator are the dominant resonant, while serial $L_{l} C_{l}$ resonator the inductor $L$ is invalidated. So, the filter can be reduced to two parallel resonators separated from one another by a transmission line with an overall length of $2 h$, as shown in Fig. 2 (c). So, the center frequency $f_{3}$ of the second band can be obtained from the formula

$$
f_{3}=\sqrt{\left(C_{2}+C_{3}\right) / C_{2} C_{3}\left(L_{2}+L_{3}\right)} / 2 \pi
$$

It should be mentioned that the equivalent circuit model of the proposed FSS is suitable not only for the normal incidence, but also for oblique incidence. The relationship between circuit parameters and physical dimensions, which depends upon different polarizations and incident angles of the incoming wave, can also be obtained from equations in [16]. For the sake of simplicity, the frequency response under the normal incidence is usually synthesized. Practically, the normal incidence frequency response is of the most important because it can directly present the performance of the FSS. Note that, because of lack of numerical investigation for the spatial couplings, the equivalent circuit model provided is useful for qualitative analysis and to further understand the operation mechanism.

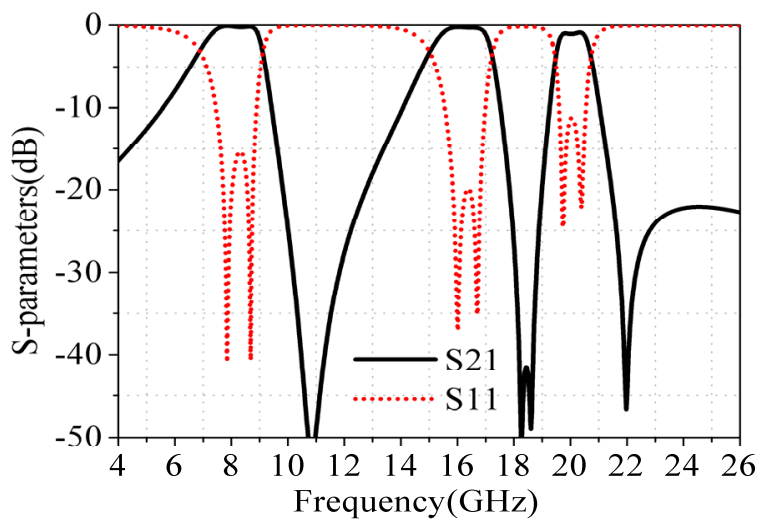

Fig.3 Simulated results of the proposed FSS under normal incidence

\section{Simulation And Measurement}

To understand the operating principle of the tri-band FSS, the frequency responses of the proposed FSS are firstly implemented using full wave electromagnetic wave simulator CST Microwave Studio. The optimized dimensions of parameters are shown in Fig.1. The transmission and reflection coefficients under normal incidence are shown in Fig.3, in which $\mathrm{S}_{21}$ denotes the transmission coefficients and $S_{11}$ represents the reflection coefficients. It is observed that there are three transmission windows centered at $8.40 \mathrm{GHz}, 15.80 \mathrm{GHz}$ and $19.88 \mathrm{GHz}$, respectively. In each band, there are two reflection zeros, which leading to a flat band response. The $-3 \mathrm{~dB}$ bandwidths of the three transmission windows are $2.04 \mathrm{GHz}, 1.72 \mathrm{GHz}$ and $1.16 \mathrm{GHz}$, respectively. In addition, there is a sharp rejection performance for these bass-bands. The transmission coefficients decrease more than $10 \mathrm{~dB}$ when frequency changes $1 \mathrm{GHz}$, on both sides to each band. Thus, the proposed FSS has high selectivity.

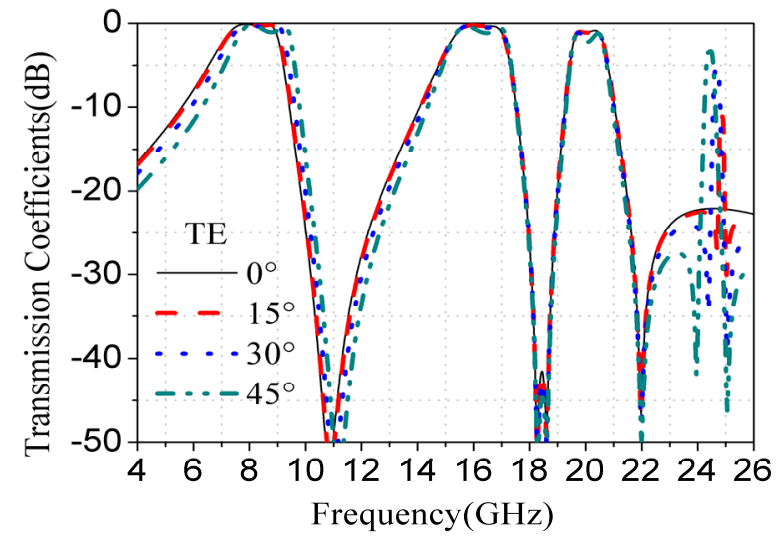

(a)TE polarization

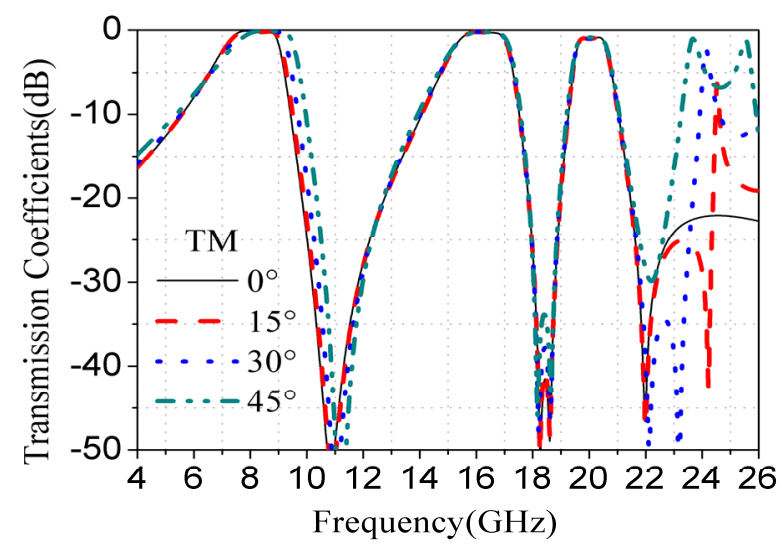

(a)TM polarization

Fig.4 Simulated results of the proposed FSS under oblique incidence with different polarizations

The transmission coefficients under oblique incident angle for TE and TM polarizations are also simulated and shown in Fig. 4. It can be seen that the proposed FSS demonstrates a stable frequency response under oblique incident angles in range of $0^{\circ}$ and $45^{\circ}$ for both polarizations, though a grating lobe occurs at higher frequency range with the incident angle increasing. In addition, when increasing the incident angle, the passband bandwidth decreases for TE polarization while increases for TM polarization. The variations of the transmission response in-band or out-of-band with respect to the incident angle are attributed to the change of wave impedance.

To further validate the simulated results, a prototype of the proposed FSS is fabricated using the printed circuit board technology (PCB) and tested utilizing a free space 
measurement method in a microwave anechoic chamber, as shown in Fig. 5. The exterior two metal layer structures are patterned on one side of two dielectric substrate of F4B-2 with thickness of $1.0 \mathrm{~mm}$. The middle layer structure is arranged on the other surface of one dielectric substrate of F4B-2. The two dielectric substrate of F4B-2 are bonded together using very thin bonding films, and thus the proposed FSS can be obtained. The relative position of metallic layers, dielectric substrate and air foam are shown in Fig.1. The dimension of the prototype is $360 \times 360 \mathrm{~mm}^{2}$, containing $72 \times 72$ elements. In microwave anechoic chamber, the FSS is placed on a rotated platform and two standard horn antennas operating in $\mathrm{C}, \mathrm{X}, \mathrm{Ku}, \mathrm{K}$-band are placed at distance of $1.2 \mathrm{~m}$ from each side of the FSS panel to ensure that the FSS is excited with plane wave. To ensure the accuracy of the experiment, the measurement was taken by two steps: firstly, the transmission coefficient without the FSS prototype is measured; secondly, the FSS prototype is placed between the two antennas and the transmission coefficient is measured. At last, the second tested results are calibrated using the results measured in first step. Fig. 6 shows the measured transmission performance of the FSS under normal incidence, and the simulated results are also given for comparison. Apparently, the measured frequency response agrees well with the simulated result.

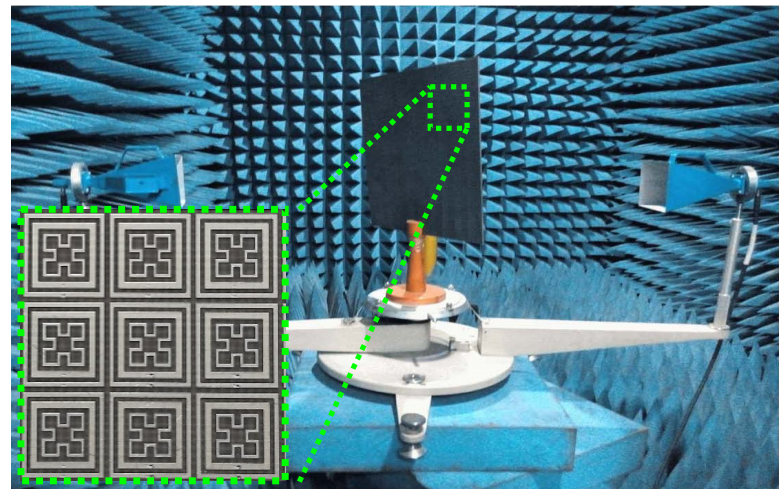

Fig.5 Measured system in microwave chamber.

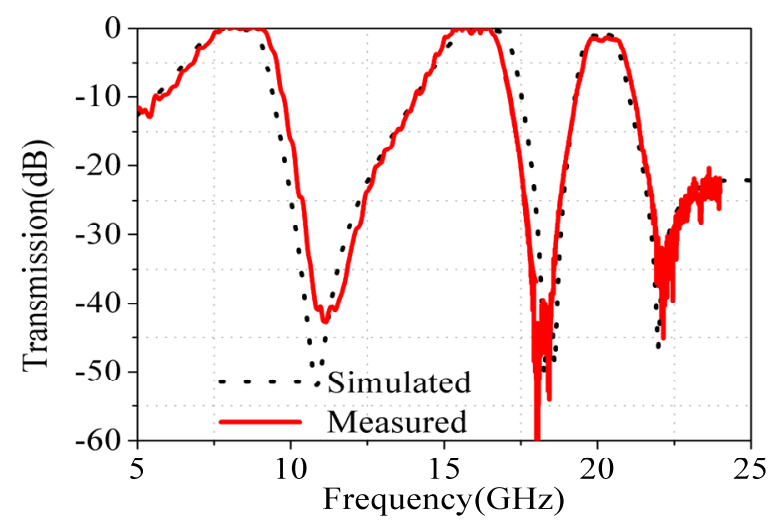

Fig.6 Measured results of the proposed FSS prototype under normal incidence

\section{Conclusions}

In this paper, a tri-band frequency selective surface with second-order response is designed and synthesized. The proposed FSS provides three transmission windows that are separated from one another by two transmission zeros, leading to a highly selective response. Each pass-band contains two transmission poles, which results in a broad and flat band performance. The simulation is implemented using full wave electromagnetic wave simulator CST Microwave Studio, and the operating principle is also described. Furthermore, the FSS has the merits of polarization insensitivity and incident angle stability.

\section{References}

1. Wu T. K.: 'Frequency Selective Surfaces and Grid Arrays' (Wiley, New York, 1995)

2. Munk B.A.: 'Frequency selective surfaces: theory and design' (Wiley, New York, 2000)

3. Johnson J. M., Rahmat-Samii Y.: 'Genetic algorithms in engineering electromagnetics', IEEE Antennas Propagat. Mag., 1997, 39, pp. 7-25

4. Charkravarty S., Mittra R., Williams N. R.: 'On the application of the microgenetic algorithm to the design of broad-band microwave absorbers comprising frequency-selective surfaces embedded in multilayered dielectric media', IEEE Trans. Microwave Theory Tech., 2001, 49, pp. 1050-1059

5. Manara G., Monorchio A., Mittra R.: 'Frequency selective surface design based on genetic algorithm', Electron. Lett., 1999, 36, (17), pp. 1400-1401

6. Wu T. K., Lee S. W.: 'Multiband frequency selective surface with multi-ring patch elements', IEEE Trans. Antennas Propag., 1994, 42(11), pp.1484-1490

7. Huang J., Wu T. K., Lee S. H.: 'Tri-band frequency selective surface with circular ring elements'. IEEE Trans. Antennas Propag., 1994, 42(2), pp. 166-175

8. Romeu J., Rahmat-Samii Y.: 'Fractal FSS: A novel dual-band frequency selective surface', IEEE Trans. Antennas Propag., 2000, 48(7), pp. 1097-1105

9. Werner D. H., Lee D.: 'Design of dual-polarised multiband frequency selective surfaces using fractal elements', Electron. Lett., 2000, 36(6), pp. 487-488

10. Hu X.D., Zhou X.L., Wu L.S., Zhou L., Yin W.Y.: 'A miniaturized dual-band frequency selective surface (FSS) with closed loop and its complementary pattern', IEEE Antennas Wireless Propag. Lett., 2009, 8, pp. 1374-1377

11. M. B. Yan, et.al.: 'A Miniaturized Dual-Band FSS With Second-Order Response and Large Band Separation', IEEE Antennas Wireless Propag. Lett., 2015, Vol.14, pp. 1602-1605

12. Bayatpur F., Sarabandi K.: 'Multi-pole spatial filters using metamaterial based miniaturized-element frequency-selective surfaces', IEEE Trans. Microw. Theory Tech., 2008, 56(12), pp. 2742-2747

13. Salehi M., Behdad N.: 'A Second-Order Dual X-/KaBand Frequency Selective Surface: 'IEEE Microw. Wireless Compon. Lett., 2008, 18(12), pp. 785-787

14. Luo G. Q., Hong W., Hao Z.C., Liu B., Li W. D., Chen 
J. X., Zhou H. X., Wu K.: 'Theory and Experiment of Novel Frequency Selective Surface Based on Substrate Integrated Waveguide Technology', IEEE Trans. Antennas Propag., 2005, 53, pp. 4035-4043

15. B. Li, Shen Z.X.: 'Three Dimensional band-pass frequency Selective structures with multiple transmission zeros', IEEE Trans. Microw. Theory Tech., 2013, 61(10), pp. 3578-3589

16. M. B. Yan, J. F. Wang, S. B. Qu, H. Ma, M. D. Feng, Y. Q. Pang, J. Q. Zhang, L. Zheng, "A Tri-Band, Highly Selective, Bandpass FSS Uing Cascaded Multilayer Loop Arrays", IEEE Trans. Antennas Propag., Vol. 64, pp. 2046-2049, 2016. 